\title{
Transatlantica
}

Revue d'études américaines. American Studies Journal

$1 \mid 2002$

Jeune République

\section{Henry Clay et la South American Question.}

La campagne au Congrès pour la reconnaissance des républiques

hispano-américaines (1818)

\section{Monica Henry}

\section{(2) OpenEdition}

Journals

Édition électronique

URL : http://journals.openedition.org/transatlantica/404

DOI : $10.4000 /$ transatlantica.404

ISSN : 1765-2766

Éditeur

AFEA

\section{Référence électronique}

Monica Henry, « Henry Clay et la South American Question. », Transatlantica [En ligne], 1 | 2002, mis en ligne le 23 mars 2006, consulté le 29 avril 2021. URL : http://journals.openedition.org/transatlantica/ 404 ; DOI : https://doi.org/10.4000/transatlantica.404

Ce document a été généré automatiquement le 29 avril 2021

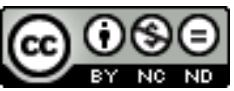

Transatlantica - Revue d'études américaines est mis à disposition selon les termes de la licence Creative Commons Attribution - Pas d'Utilisation Commerciale - Pas de Modification 4.0 International. 


\section{Henry Clay et la South American Question.}

La campagne au Congrès pour la reconnaissance des républiques hispano-américaines (1818)

\section{Monica Henry}

1 Cet article s'inscrit dans un travail de thèse plus large qui analyse à travers des sources variées les rapports entre la jeune République américaine et les républiques émergentes d'Amérique du Sud, à l'époque de l'indépendance de ces dernières. L'historiographie classique de la question s'est surtout penchée sur le dirigeant américain dont les idées ont forgé les positions de son pays, à savoir John Quincy Adams. Moins nombreux sont les historiens qui ont cherché à connaître les courants d'opinion minoritaires, ceux qui tentaient de faire valoir d'autres idées, peut-être plus généreuses ou plus ambitieuses pour l'avenir du continent américain. On trouvera donc ici retracé un débat du Congrès, en mars 1818, où Henry Clay et ses amis se battirent pour obtenir une reconnaissance rapide des nouvelles républiques voisines, sans succès. Leurs idées et leurs arguments méritent cependant d'être rappelés.

Lorsque James Monroe devient président en avril 1817, son gouvernement doit consacrer toute son attention aux relations entre les États-Unis et l'empire espagnol. Le cabinet, le département d'État, tout comme le Congrès, vont s'occuper longuement de discuter des incursions du général Jackson dans les territoires sous contrôle espagnol, de l'occupation par l'armée américaine de l'île d'Amélia, des négociations entre le secrétaire d'État John Quincy Adams et le ministre espagnol Luis de Onis pour résoudre les conflits territoriaux, notamment sur la frontière sud des États-Unis, des tensions causées par les actions des corsaires qui agissaient depuis les ports américains contre des bateaux espagnols, et enfin plus généralement des révolutions et des guerres d'indépendance en Amérique espagnole.

3 En juillet 1817, l'envoyé du gouvernement de Buenos Aires, Don Manuel Hermenegildo de Aguirre, arrive aux États-Unis et, dans un entretien avec John Quincy Adams, demande la reconnaissance de son gouvernement. Il échoue dans sa mission mais il réussit, en revanche, à déclencher le débat sur la South American Question (Annals of 
Congress $15^{\text {ème }}$ Congrès, 1 ère Session). En effet, dès les premiers mois de 1818 , sous l'impulsion de Henry Clay et de ses amis, représentants du Kentucky, pour la plupart, mais aussi d'autres États, une campagne en faveur de la reconnaissance des républiques hispano-américaines s'intensifie au Congrès. Le 24 mars 1818, Clay demande à la Chambre de considérer l'octroi de 18000 dollars pour : « one year's salary, and an outfit to a minister to the United Provinces of the Rio de la Plata » (aujourd'hui l'Argentine) (Annals 24 mars).

4 Voici un moment important dans l'élaboration et mise en place de la politique « hispano-américaine » car, d'une part, c'est la première fois que le Congrès consacre autant de temps à débattre et à critiquer la politique officielle à l'égard des gouvernements révolutionnaires. D'autre part, la reconnaissance - trop hâtive pour les uns, trop tardive pour les autres - du gouvernement de Buenos Aires, qui se trouve quand même loin des frontières américaines, représente un enjeu considérable pour cette nation américaine qui se veut une puissance sur la scène internationale.

5 A l'époque, les adversaires de Clay, notamment John Quincy Adams, pensaient, voire soupçonnaient, que le Speaker de la Chambre-motivé par ses ambitions présidentielles - en profitait pour lancer une attaque contre l'exécutif (Adams 186). Aujourd'hui, on analyse plutôt ces débats comme la preuve de tensions existantes entre les différents intérêts régionaux, l'ouest étant représenté par Henry Clay (Weeks 85-104). Sans réfuter ces analyses, il nous semble qu'à travers ces débats, on voit surgir, chez les Américains, deux visions différentes de la place des États-Unis sur le continent américain d'après les révolutions et indépendances hispano-américaines. L'une est présentée par Henry Clay, l'autre par ses opposants, groupés autour d'un représentant de l'État de Géorgie, John Forsyth.

Dans un discours de trois heures, Henry Clay souligne que le droit de reconnaître l'indépendance de facto d'une autre nation revient à tout État souverain. Ce droit, en l'occurrence, est compatible avec la politique de neutralité adoptée par le gouvernement américain vis-à-vis de la guerre opposant l'Espagne à ses colonies, car reconnaissance ne signifiait pas participation dans cette guerre civile (Annals 24 mars). Dans le débat qui suivit, la question fut donc de savoir si les États-Unis transgressaient les lois de neutralité s'ils reconnaissaient le gouvernement de Buenos Aires. Pour pouvoir y répondre, les représentants se posèrent trois questions: (1) Pourquoi reconnaître les Provinces Unies ? (2) Quelles sont les conditions nécessaires pour pouvoir les reconnaitre? (3) Quels seraient alors les risques encourus?

1 - Les raisons

7 Clay affirme que les États-Unis ont tout intérêt à avoir des voisins indépendants. Le représentant de Louisiane, Robertson, explique que le libre commerce permettrait aux bateaux américains de transporter des matières premières des anciennes colonies vers l'Europe ainsi que des produits manufacturés européens et américains vers les ports hispano-américains. La demande croissante de produits américains qui s'ensuivrait aurait aussi un impact sur l'agriculture des États-Unis. Avec l'augmentation de la demande de matières premières pour l'industrie manufacturière américaine, le prix de ces matières premières augmentera. Robertson démontre ainsi l'interconnexion des trois activités de l'économie américaine et leur lien avec l'économie mondiale (Annals 26 mars).

8 Le principal opposant de Henry Clay, John Forsyth, ne croit pas, au contraire, qu'en se lançant dans le commerce avec l'Amérique latine, l'industrie manufacturière puisse un 
jour être plus rentable que l'agriculture. De plus, les États-Unis se verraient obligés de concurrencer les entreprises navales hispano-américaines qui allaient se créer après l'indépendance, une donnée que le Speaker ne prend pas en compte, accuse Forsyth (Annals 25 mars). Mais en fin de compte, rétorque Clay, pouvoir témoigner de la sympathie à ces frères qui se battent pour leur liberté est toujours plus important que toutes ces considérations commerciales.

9 Selon lui, les nouvelles républiques hispano-américaines vont devenir le grand sanctuaire de la liberté et l'asile contre toute persécution, comme le sont déjà les États-Unis. Elles seront animées par l'esprit américain et guidées par la politique américaine - Clay y emploie le terme "américain " pour faire référence à tout le continent américain (Annals 24 mars). Son collègue de Virginie, Floyd, y voit l'occasion de systématiser une politique pour tout le continent et Robertson de consolider la position des États-Unis dans l'hémisphère occidental. Si les États-Unis n'aident pas leurs amis et alliés naturels, Johnson (du Kentucky) craint que les nouvelles républiques ne se tournent vers d'autres puissances, à savoir les nations européennes (Annals 26 mars). Le danger, d'une part, de voir les Hispano-américains basculer dans le champ monarchique et d'autre part, de créer une cause d'hostilité, à l'avenir, entre Hispano-américains et Américains du Nord était réel, d'après les défenseurs de la cause révolutionnaire.

10 Afin de se protéger contre ce possible danger, Smyth, de Virginie, invoque le droit qu'a toute nation de se défendre contre des mauvais voisins en refusant, en l'occurrence, de les reconnaitre (Annals 26 mars). Et Forsyth demande, lui, pourquoi on mettrait en péril le bonheur et la sécurité des citoyens américains pour essayer d'harmoniser le système politique des États-Unis avec ces républiques sœurs (Annals 25 mars).

11 En somme, les deux camps s'appuient sur des raisons d'intérêt économique et de sécurité, que ce soit pour promouvoir ou pour condamner l'envoi d'un ministre, et par voie de conséquence, la reconnaissance du gouvernement de Buenos Aires.

2 - Les conditions

12 La Constitution des États-Unis prévoit, d'après Henry Clay, trois modes possibles de reconnaissance: (1) l'exécutif reçoit un ministre; (2) l'exécutif envoie un ministre américain ; ou bien (3) la Chambre des représentants a le droit de reconnaître un autre gouvernement afin de réguler le commerce un droit constitutionnel qui revient au Congrès. Par ailleurs, poursuit Clay, le gouvernement américain s'est toujours abstenu de se prononcer en faveur d'un souverain de droit et contre un souverain de fait. Washington avait déjà envoyé un ministre au Brésil, gouverné par un monarque, pourquoi donc, se demande Clay, ne pas envoyer un ministre dans une république, comme celle du Rio de la Plata? Depuis la révolution de 1810, à Buenos Aires, la stabilité et l'ordre se sont maintenus dans ce coin du monde, preuve suffisante, estime-t-il, qu'il s'agit d'un vrai gouvernement. Et il conclut qu'étant donné l'existence d'un gouvernement (bien) établi en Amérique espagnole, les États-Unis ont l'obligation morale et politique de le reconnaître (Annals 28 mars).

Pour Forsyth, au contraire, les États-Unis ont le droit de reconnaitre les Province Unies du Rio de la Plata mais il n'en ont pas nécessairement l'obligation, d'autant plus que les nouvelles provenant du continent sud sur la guerre sanglante qui continue d'opposer les forces révolutionnaires aux royalistes ne sont nullement rassurantes. L'indépendance politique des Hispano-Américains leur a été, en quelque sorte, imposée lorsque le roi Ferdinand d'Espagne a été fait prisonnier par l'armée de Napoléon, tandis 
qu'aux États-Unis, les Américains se sont battus pour l'obtenir. Ce fut $a$ war of the people, dit Forsyth avec insistance, dans laquelle le pouvoir civil a toujours contrôlé le pouvoir militaire (Annals 25 mars). Selon un des représentants du Mississippi, Poindexter, le chef du gouvernement des Provinces unies, le militaire de carrière J. M. de Pueyrredon, n'a pas donné de preuves suffisantes de son attachement aux principes fondamentaux d'un gouvernement libre ; il gouverne, si l'on en croit Poindexter, d'une main de fer (Annals 28 mars).

La contre-attaque ne se fait pas attendre. Quelle révolution a commencé par assurer les libertés civiles? se demande Robertson. Et il répond que ce ne fut certainement pas la Révolution américaine. Celle-ci n'a-t-elle pas été aussi sanglante? s'interroge Floyd ${ }^{1}$. L'indépendance n'était pas non plus l'objectif premier de la Révolution américaine, conteste Clay².

Autrement dit, les conditions pour pouvoir reconnaître le gouvernement de Buenos Aires existent pour les uns, n'existent pas pour les autres selon les interprétations divergentes des informations qui arrivaient aux États-Unis et les conceptions différentes de la Révolution américaine qu’a chaque représentant au Congrès. Mais pour Holmes, un représentant du Massachusetts, toutes ces considérations n'ont aucune importance, et il conclut son intervention par un appel lyrique à la solidarité :

This nation now stands alone, the only established Republic on earth, like a solitary rock in the ocean, where the storms of tyranny have burst upon its brow, and the bellows of faction broke harmless at its base. Will it not then be a source of consolation that we can hail one Republic as a sister, take her by the hand, and encourage her in her advance to freedom? (Annals 27 mars)

Pourtant, cette expression de solidarité ne convainc pas vraiment les plus sceptiques, qui cherchent plutôt à mesurer les conséquences de l'adoption de la motion proposée par Henry Clay.

3 - Les conséquences

16 Nelson, représentant de Virginie, craint une division au sein de la classe politique entre les soi-disant amis de la liberté et les indifférents à la lutte révolutionnaire et, par conséquence, la destruction de l'harmonie existante de «l'ère de bons sentiments ». Cette division déplorable serait causée par des facteurs extérieurs ; on retournerait à la vieille histoire, horrible selon lui, de l'influence étrangère sur les affaires intérieures américaines (Annals 27 mars). Néanmoins un danger plus imminent perturbe les esprits : si les États-Unis reconnaissaient le gouvernement de Buenos Aires, l'Espagne réagirait-elle? Pendant les débats, beaucoup de temps est consacré à ce point délicat, c'est-à-dire au risque de guerre contre l'empire espagnol, avec lequel l'Amérique était encore en litige. Les négociations entre John Quincy Adams et le ministre espagnol Luis de Onis n'étaient pas encore achevées: on sait qu'elles devaient conduire, en 1819, à l'intégration des Florides dans le territoire américain et à la démarcation de la frontière entre les États-Unis et l'empire espagnol de l'Atlantique jusqu'au Pacifique.

Henry Clay était convaincu que l'Espagne était trop fatiguée et sans ressources pour pouvoir s'engager dans encore un autre conflit armé (Annals 24 mars). De plus, comme le signale Robertson (de Louisiane), l'Espagne avait déjà été provoquée - en ce moment même l'armée américaine se trouvait sur le territoire espagnol en train de chasser des Indiens, dit-il - et elle n'avait réagi qu'en protestant par l'intermédiaire de son ministre à Washington (Annals 25 mars). Mais au-delà de la conjoncture, une question de principe se posait : contre l'Espagne, existait-t-il une guerre juste ? Clay ne le pensait 
pas car les États-Unis, en reconnaissant la nouvelle république, ne violaient pas les lois de neutralité.

D'après la loi des nations (en fait le droit international, tel qu'on le qualifiait à l'époque), l'Espagne ne serait pas fondée à déclarer la guerre aux États-Unis, convint Forsyth. Mais pour l'adversaire de Clay, il s'agissait plus d'une question d'usage que de principes. Si l'Espagne, par exemple, décidait de reconnaître l'indépendance de la Louisiane, les États-Unis ne considéraient-ils pas cette action comme un motif justifiant la guerre (Annals 25 mars)?

Et face à l'éventualité d'une guerre contre l'Espagne, quelle serait l'attitude de l'Angleterre, se demandaient, avec anxiété, tous les représentants ? Serait-elle prête à aider son allié, l'Espagne, ou, au contraire, s'en abstiendrait-elle afin de ne pas mettre en péril ses intérêts commerciaux dans la région?

20 En fait, la Chambre était en train de débattre d'une politique étrangère américaine qui suivrait davantage la politique européenne attentiste envers l'indépendance des colonies espagnoles. Ou bien d'une politique américaine plus indépendante, qui prendrait l'initiative face à une Europe hésitante, voire réticente, et lente à porter secours aux Espagnols.

Conclusion

21 Le résultat final fut le suivant: 45 votes pour, 115 votes contre. Le Speaker de la Chambre fut sévèrement battu et John Quincy Adams s'en réjouit. L'amendement proposé par Henry Clay ne fut donc pas adopté. Résultat assez étonnant vu l'éloquence et la variété d'arguments déployés pour convaincre une majorité silencieuse qui, de toute évidence, ne s'est pas laissée impressionner par cette effervescence prorévolutionnaire et qui a préféré rester prudente.

Néanmoins, on peut considérer, grâce à cette lecture attentive des débats, que la vision de Clay et de ses partisans, en apparence idéaliste, était pleine de sagesse. Ces orateurs présentent la possibilité d'un continent américain plus intégré, où s'établiraient des relations plus fluides, plus harmonieuses peut-être, entre le sud et le nord. Ils savent que les Hispano-Américains sont et resteront pour toujours des voisins à prendre en compte.

Le groupe de Forsyth, en revanche, se concentre sur les préoccupations plus concrètes, immédiates et pressantes du pays. Ces soi-disant «réalistes » ne prévoient pas de partager leur avenir avec les voisins du sud, c'est à dire qu'ils n'envisagent pas un avenir continental.

24 Tout examen approfondi des débats législatifs portant sur ce sujet nous conduit inévitablement à une question majeure. De laquelle de ces deux visions des relations entre le Nord et le Sud est tributaire la doctrine Monroe de 1823 ?

\section{BIBLIOGRAPHIE}

Sources primaires 
Annals of the Congress of the United States. $15^{\text {ème }}$ Congrès, 1ère session, 24-28 mars. Washington D. C., 1854-1855.

Adams, John Quincy. Memoirs of John Quincy Adams. Ed. Charles Francis Adams. New York: AMS Press, 1970.

Adams, John Quincy. The Writings of John Quincy Adams. Ed. Worthigton Chauncey Ford. New York, 1913-1917.

Clay, Henry. Life and Speeches of Henry Clay. Ed. Daniel Mallory. New York: Van Amringe and Bixby, 1844.

Clay, Henry. The Papers of Henry Clay. Ed. James F. Hopkins. Lexington: University of Kentucky Press, 1961.

Niles' Weekly Register, Baltimore, 1811-1849.

Sources secondaires

Baxter, Maurice G. Henry Clay and the American System. Lexington: The University Press of Kentucky, 1995.

Bemis, Samuel Flagg. John Quincy Adams and the Foundations of American Foreign Policy. New York: Alfred A. Knopf, 1956.

Coulter, E. Merton. « The Genesis of Henry Clay's American System ». South Atlantic Review 25 (January 1926): 45-54.

Cummins, Light T. «John Quincy Adams and Latin American Nationalism ». Revista de Historia de América 86 (julio-diciembre 1978): 221-231.

Griffin, Charles C. The United States and the Disruption of the Spanish Empire, 1810-1822. New York: Columbia University Press, 1937.

Dangerfield, George. The Era of Good Feelings. New York: Harcourt, Brace and Company, Inc., 1952. Hoskins, Halford. « The Hispanic American Policy of Henry Clay, 1816-1828 ». Hispanic American Historical Review 7 (November 1927) : 460-478.

LaFeber, Walter, ed. John Quincy Adams and American Continental Empire. Letters, Papers and Speeches. Chicago: Quadrangle Books, 1965.

Lang, Daniel G. Foreign Policy in the Early Republic. The Law of Nations and the Balance of Power. Baton Rouge: Louisiana State University Press, 1985.

Langley, Lester D. The Americas in the Age of Revolution, 1750-1850. New Haven: Yale University Press, 1996.

Lewis Jr., James E. The American Union and the Problem of Neighborhood. The United States and the Collapse of the Spanish Empire, 1783-1829. Chapel Hill: The University of North Carolina Press, 1998.

Remini, Robert V. Henry Clay. Statesman for the Union. New York: W. W. Norton, 1991.

Robertson, William Spence. « The Recognition of the Hispanic American Nations by the United States », Hispanic American Historical Review 1 (August 1918): 239-269.

Robledo, Alfonso. « Elogio a Henry Clay ». Hispanic American Historical Review 6 (November 1926): 199-204.

Watson, Harry L. Andrew Jackson vs. Henry Clay. Democracy and Development in Antebellum America. Boston: Bedford/St.Martin's, 1998. 
Weeks, William Earl. John Quincy Adams and American Global Empire. Lexington: The University Press of Kentucky, 1992.

Whitaker, Arthur Preston. The United States and the Independence of Latin America (1941). New York: Russell \& Russell, 1962.

\section{NOTES}

1. Ibid., 26 mars 1818.

2. Ibid., 28 mars 1818.

\section{RÉSUMÉS}

In March 1818 the Speaker of the House of Representatives Henry Clay raised the question of recognition of the new Spanish-American Republics in the House by presenting a resolution making an appropriation for a minister to Buenos Aires. This article argues that beyond the debate that took place in Congress about whether to recognize or not the new governments in Spanish America, what really divided the Representatives was opposing visions of future relations between North and South America in the post-revolutionary period.

\section{INDEX}

Keywords : Henry Clay, Congress, Spanish empire, independence, recognition, South America Mots-clés : Congrès, empire espagnol, indépendance, reconnaissance, Amérique du Sud

\section{AUTEUR}

\section{MONICA HENRY}

Monica Henry prépare un doctorat à l'Institut d'anglais Charles-V (Université Paris 7) sous la direction de Marie-Jeanne Rossignol sur « Les relations entre les Etats-Unis et les nouvelles républiques hispano-américaines, 1810-1826». 